\title{
The Prevalence and Subtypes of Young Onset Dementia in Central Norway: A Population-Based Study
}

[Journal of Alzheimer's Disease, 69(2) (2019), 479-487, DOI 10.3233/JAD-181223]

https://content.iospress.com/articles/journal-of-alzheimers-disease/jad181223

On page 1 and 6 incorrect values have been given. The correct values are given below.

On page 1, in line 2 of the Results section of the Abstract: 76.3 per 100,000 should be 85.5 per 100,000.

On page 1, in line 3 of the Results section of the Abstract: 163.1 per 100,000 should be 143.1 per 100,000.

On page 5, line 10 of the Discussion section: 76.3 per 100000 should be 85.5 per 100000 .

On pages 484 and 485: Tables 4-6 contain incorrect values. The correct Tables 4-6 are listed below.

Table 4

Age and gender-specific prevalence rates in the study population

\begin{tabular}{|c|c|c|c|c|c|c|c|c|c|c|c|}
\hline \multirow{2}{*}{\multicolumn{3}{|c|}{ Population }} & \multicolumn{9}{|c|}{ All causes of dementia } \\
\hline & & & \multicolumn{3}{|c|}{ All } & \multicolumn{3}{|c|}{ Male } & \multicolumn{3}{|c|}{ Female } \\
\hline Age range & Male (n) & Female (n) & $n$ & Rate* $^{*}$ & $95 \% \mathrm{CI}$ & $\mathrm{n}$ & Rate* $^{*}$ & $95 \% \mathrm{CI}$ & $\mathrm{n}$ & Rate* & $95 \% \mathrm{CI}$ \\
\hline $30-34$ & 14955 & 13956 & 2 & 6.9 & $(1.0-25.0)$ & 2 & 13.4 & $(1.6-48.3)$ & 0 & - & - \\
\hline $35-39$ & 14451 & 13145 & 1 & 3.6 & $(1.0-20.2)$ & 0 & - & - & 1 & 7.6 & $(1.0-42.4)$ \\
\hline $40-44$ & 15656 & 14683 & 6 & 19.8 & $(7.3-43.0)$ & 5 & 31.9 & $(10.4-74.5)$ & 1 & 6.8 & $(1.0-38.0)$ \\
\hline $45-49$ & 16094 & 15507 & 7 & 22.2 & $(8.9-45.6)$ & 5 & 31.1 & $(10.1-72.5)$ & 2 & 12.9 & $(1.6-46.6)$ \\
\hline $50-54$ & 14908 & 14146 & 27 & 92.9 & $(61.3-135.2)$ & 11 & 73.8 & $(36.8-132.0)$ & 16 & 113.1 & $(64.7-183.6)$ \\
\hline $55-59$ & 13762 & 13199 & 44 & 163.2 & (118.6-219.0) & 21 & 152.6 & $(94.5-233.2)$ & 23 & 174.3 & $(110.5-261.4)$ \\
\hline $60-64$ & 12830 & 12732 & 84 & 328.6 & $(262.2-406.7)$ & 42 & 327.4 & $(236.0-442.2)$ & 42 & 329.9 & $(237.8-445.6)$ \\
\hline $30-44$ & 45062 & 41784 & 9 & 10.4 & $(4.7-19.7)$ & 7 & 15.5 & $(6.2-32.0)$ & 2 & 4.8 & $(1.0-17.3)$ \\
\hline $30-64$ & 102656 & 97368 & 171 & 85.5 & (73.2-99.3) & 86 & 83.8 & $(67.0-103.5)$ & 85 & 87.3 & $(69.7-107.9)$ \\
\hline $45-64$ & 57594 & 55584 & 162 & 143.1 & $(122.0-166.9)$ & 79 & 137.2 & $(108.6-170.9)$ & 83 & 149.3 & (119.0-185.1) \\
\hline
\end{tabular}

* Rate per 100000 people at risk. 
Table 5

Age specific prevalence rates for the most common causes of YOD

\begin{tabular}{|c|c|c|c|c|c|c|c|c|c|c|c|c|}
\hline \multirow[b]{2}{*}{ Age range } & \multicolumn{2}{|c|}{ Alzheimer's disease } & \multicolumn{3}{|c|}{ HD dementia } & \multicolumn{2}{|c|}{ Alcohol related dementia } & \multicolumn{3}{|c|}{ Vascular dementia } & \multicolumn{2}{|c|}{ Frontotemporal dementia } \\
\hline & n Rate* & $95 \% \mathrm{CI}$ & & N Rate* & $95 \% \mathrm{CI}$ & n Rate* & $95 \% \mathrm{CI}$ & $\mathrm{n}$ & Rate* $^{*}$ & $95 \% \mathrm{CI}$ & n Rate* & $95 \% \mathrm{CI}$ \\
\hline $35-39$ & & & & & & & & 1 & 3.6 & $(1.0-20.2)$ & & \\
\hline $40-44$ & & & 5 & 516.5 & $(5.4-38.5)$ & & & 1 & 3.3 & $(1.0-18.4)$ & & \\
\hline $45-49$ & & & 3 & 39.5 & $(2.0-27.7)$ & & & 1 & 3.2 & $(1.0-17.6)$ & & \\
\hline $50-54$ & $\begin{array}{ll}6 & 20.7\end{array}$ & $(7.6-44.9)$ & 4 & +13.8 & $(3.8-35.2)$ & 26.9 & $(1.0-24.9)$ & 1 & 3.4 & $(1.0-19.2)$ & 13.4 & $(1.0-19.2)$ \\
\hline $55-59$ & 1555.6 & $(31.1-91.7)$ & 3 & 311.1 & $(2.3-32.5)$ & 27.4 & $(1.0-26.8)$ & 3 & 11.1 & $(2.3-32.5)$ & 726.0 & $(10.4-53.5)$ \\
\hline $60-64$ & 53207.3 & $(155.3-271.1)$ & 5 & 19.6 & $(6.4-45.6)$ & $\begin{array}{ll}7 & 27.4\end{array}$ & $(11.0-56.4)$ & 4 & 15.6 & $(4.3-40.1)$ & $4 \quad 15.6$ & $(4.3-40.1)$ \\
\hline $30-64$ & 7437.0 & $(29.1-46.4)$ & 21 & 110.5 & $(6.5-16.0)$ & 115.5 & $(2.7-9.8)$ & 11 & 5.5 & $(2.7-9.8)$ & 126.0 & $(3.1-10.5)$ \\
\hline $45-64$ & $74 \quad 65.4$ & $(51.3-82.1)$ & 15 & 513.3 & (7.4-21.9) & 119.7 & $(4.9-17.4)$ & 9 & 8.0 & $(3.6-15.1)$ & 1210.6 & $(5.5-18.5)$ \\
\hline
\end{tabular}

*Rate per 100000 people at risk.

Table 6

Prevalence rates per 100,000 persons at risk of dementia and subtypes in various population-based studies

\begin{tabular}{|c|c|c|c|c|c|c|c|c|c|c|}
\hline \multicolumn{11}{|c|}{ All dementia } \\
\hline & \multicolumn{2}{|c|}{$\begin{array}{c}\text { Norway } \\
\text { (Current } \\
\text { study) }\end{array}$} & \multicolumn{2}{|c|}{$\begin{array}{c}\text { Australia } \\
\text { (Whithall } \\
\text { et al., 2014) }\end{array}$} & \multicolumn{2}{|c|}{$\begin{array}{c}\text { Japan } \\
\text { (Ikejima } \\
\text { et al., 2009) }\end{array}$} & \multicolumn{2}{|c|}{$\begin{array}{l}\text { England } \\
\text { (Harvey } \\
\text { et. Al 2003) }\end{array}$} & \multicolumn{2}{|c|}{$\begin{array}{c}\text { England } \\
\text { (Ratnavalli } \\
\text { et al., 2002) }\end{array}$} \\
\hline \multicolumn{11}{|l|}{ Age: } \\
\hline $50-54$ & \multicolumn{2}{|c|}{92.9} & \multicolumn{2}{|c|}{102.7} & \multicolumn{2}{|c|}{59.0} & \multicolumn{2}{|c|}{62.5} & \multicolumn{2}{|c|}{ - } \\
\hline $55-59$ & \multicolumn{2}{|c|}{163.2} & \multicolumn{2}{|c|}{131.2} & \multicolumn{2}{|c|}{94.3} & \multicolumn{2}{|c|}{152.1} & \multicolumn{2}{|c|}{-} \\
\hline $60-64$ & \multicolumn{2}{|c|}{328.6} & \multicolumn{2}{|c|}{265.2} & \multicolumn{2}{|c|}{163.3} & \multicolumn{2}{|c|}{166.3} & \multicolumn{2}{|c|}{ - } \\
\hline $30-64$ & \multicolumn{2}{|c|}{85.5} & \multicolumn{2}{|c|}{68.2} & \multicolumn{2}{|c|}{$51.7^{*}$} & \multicolumn{2}{|c|}{54.0} & \multicolumn{2}{|c|}{ - } \\
\hline $45-64$ & \multicolumn{2}{|c|}{143.1} & & & \multicolumn{2}{|c|}{83.3} & \multicolumn{2}{|c|}{98.1} & \multicolumn{2}{|c|}{81.0} \\
\hline \multicolumn{11}{|c|}{ Dementia subtypes } \\
\hline & $\begin{array}{l}\text { Norway } \\
\text { (Current } \\
\text { study) }\end{array}$ & $\begin{array}{c}\text { Australia } \\
\text { (Whithall } \\
\text { et al., } \\
\text { 2014) }\end{array}$ & $\begin{array}{c}\text { Japan } \\
\text { (Ikejima } \\
\text { et al., } \\
\text { 2009) }\end{array}$ & $\begin{array}{c}\text { England } \\
\text { (Harvey } \\
\text { et. Al } \\
2003 \text { ) }\end{array}$ & $\begin{array}{c}\text { England } \\
\text { (Ratnavalli } \\
\text { et al., } \\
\text { 2002) }\end{array}$ & $\begin{array}{c}\text { Norway } \\
\text { (Current } \\
\text { study) }\end{array}$ & $\begin{array}{c}\text { Australia } \\
\text { (Whithall } \\
\text { et al., } \\
\text { 2014) }\end{array}$ & $\begin{array}{c}\text { Japan } \\
\text { (Ikejima } \\
\text { et al., } \\
\text { 2009) }\end{array}$ & $\begin{array}{c}\text { England } \\
\text { (Harvey } \\
\text { et. Al } \\
2003 \text { ) }\end{array}$ & $\begin{array}{c}\text { England } \\
\text { (Ratnavalli } \\
\text { et al., } \\
\text { 2002) }\end{array}$ \\
\hline & & & $\mathrm{AD}$ & & & & & $\mathrm{VaD}$ & & \\
\hline Age: & & & & & & & & & & \\
\hline $50-54$ & 20.7 & 6.4 & 9.8 & 16.4 & - & 3.4 & 6.4 & 22.9 & 6.6 & - \\
\hline $55-59$ & 55.6 & 13.1 & 28.0 & 50.7 & - & 11.1 & 13.1 & 42.2 & 32.6 & - \\
\hline $60-64$ & 207.3 & 74.6 & 49.5 & 77.3 & - & 15.6 & 49.7 & 78.4 & 38.7 & - \\
\hline $30-64$ & 37.0 & 9.3 & $13.4^{*}$ & 17.4 & - & 5.5 & 7.7 & $10.1^{*}$ & 8.7 & - \\
\hline $45-64$ & 65.4 & 19.9 & 22.3 & 35.0 & 15.1 & 8.0 & 14.9 & 38.6 & 17.9 & 8.2 \\
\hline & & & FTD & & & & & ARD & & \\
\hline $50-54$ & 3.4 & 6.4 & 1.5 & 3.3 & - & 6.9 & 32.1 & - & 19.7 & - \\
\hline $55-59$ & 26.0 & 26.2 & 1.7 & 25.4 & - & 7.4 & 32.8 & - & 18.1 & - \\
\hline $60-64$ & 15.6 & 8.3 & 4.4 & 23.2 & - & 27.4 & 49.7 & - & 11.6 & - \\
\hline $30-64$ & 6.0 & 5.4 & $1.2^{*}$ & 7.5 & - & 5.5 & 16.3 & - & 6.6 & - \\
\hline $45-64$ & 10.6 & 11.6 & 2.0 & 15.4 & 15.1 & 9.7 & 33.1 & - & 13.6 & - \\
\hline
\end{tabular}

*Calculated. 